\title{
Effect of Varying Counterions on the Sensitivity of Electrohydrodynamic and Fast Atom Bombardment Mass Spectrometry
}

\author{
Johnny D. Reynolds and Kelsey D. Cook \\ Department of Chemistry, University of Tennessee, Knoxville, Tennessee, USA
}

\begin{abstract}
The abundance of ion pairs $\left(\mathrm{CA}^{+}\right)$relative to that of doubly charged ions $\left(\mathrm{C}^{2+}\right)$ in electrohydrodynamic (EH) mass spectra of a series of anions with a common dication in glycerol was found to increase in the order acetate $<$ nitrite $<$ chloride $<$ bromide $\approx$ nitrate < iodide < perchlorate. Correlation with enthalpies of hydration for the anions suggests that this trend reflects the solution chemistry of ion association. These spectra also reveal that solvation rather than interactions with the extracting field is more important in determining the overall $\mathrm{EH}$ mass spectrometric sensitivity to doubly charged ions. Therefore, the use of anions that promote more extensive ion pairing enhances the overall sensitivity to multiply charged ions that otherwise interact strongly with the solvent, but reduces sensitivity to singly charged ions. These observations hold in fast atom bombardment mass spectrometry, surviving the invasive effects of the primary beam. ( $J$ Am Soc Mass Spectrom 1990, 1, 233-237)
\end{abstract}

$\mathrm{T}$ The extreme impact of the choice of solvent [1-9] on the appearance of matrix-assisted desorption ionization (DI) mass spectra [10] arises from interacting effects of solvation [11, 12], ion pairing [13], surface activity $[14,15]$, mass transport $[13,16]$, and other aspects of solution chemistry. These in turn are convoluted with the effects of primary beam-induced damage intrinsic to most DI experiments [2, 3, 17-22] and aspects of gas-phase chemistry [23, 24]. Although separation of these variables is not generally feasible, an independent understanding of the effects of each would facilitate experimental design for optimum sensitivity. The object of the present study is to isolate the effects of ion pairing on sensitivity in electrohydrodynamic $(E H)$ and fast atom bombardment (FAB) mass spectrometry (MS).

Analyte ion-pairing effects have been observed previously in mass spectrometry [14, 15, 25-31]. Although Hand et al. [26] found that counterion effects can be suppressed by use of a liquid matrix, others $[14,15,25$, 27-31] have explored the use of surface-active counterions to enhance analyte sensitivity. However, such studies have not specifically assessed the extent of ion pairing and its impact on sensitivity.

On the basis of evidence of preferential sampling of preformed ions in FAB/MS and EHMS [32, 33], it can be predicted that neutralization due to ion pairing of singly charged analytes should decrease sensitivity. The situation is more complex in the case of multi-

Address reprint requests to Professor Kelsey D. Cook, Department of Chemistry, University of Tennessee, Knoxville, TN 37996-1600. ply charged ions. Partial charge reduction will decrease solvent-solute interactions, so that the overall sampling efficiency may increase with increased ion pairing, until gains are offset by neutralization. Of course, the effects of beam-induced damage and the possibility of gas-phase ion-molecule reactions subsequent to sampling cloud this picture somewhat.

Electrohydrodynamic mass spectrometry [33] provides a means of studying the effects of solution chemistry without interference from an invasive primary atom beam. In EHMS, sampling relies solely on the extracting action of an electric field at the tip of a biased capillary emitter to remove "preformed" ions from a liquid matrix. This results in characteristically simple mass spectra without fragmentation, because the desorption process adds very little, if any, internal energy to the molecule.

In EHMS, any sensitivity gains due to reduced solvation of multiply charged ions undergoing ion pairing will be offset by a corresponding decrease in interactions between the analyte ion cluster and the extracting electric field. Barring interference from gasphase chemistry, the relative importance of these offsetting trends should be evident from comparison of the degree of ion pairing and overall sensitivity for a diquaternary ammonium (diquat) salt in glycerol solutions containing anions known to vary in extent of ion pairing. Neutralization effects will be assessed from similar work with a singly charged quaternary ammonium (quat) analyte. Corresponding studies using FAB/ MS will then be used to assess whether correlation survives the invasive effects of the primary atom beam. 


\section{Experimental}

\section{Electrohydrodynamic Mass Spectrometry}

Electrohydrodynamic mass spectra were obtained with an AEI MS-902 mass spectrometer equipped with a VG Update electronics console, operating at an accelerating (emitter) potential of $8 \mathrm{kV}$, with slits fully open (resolution $\sim 400,10 \%$ valley definition) for maximum sensitivity. The design and operation of the source have been described elsewhere [33, 34]. The emitter was a $200-\mu \mathrm{m}$ i.d. platinum capillary (Hamilton). Its potential was matched to the ESA bandpass by maximizing the signal intensity of the ion at $\mathrm{m} / z=207$ ( $\left[\mathrm{Na}+\mathrm{G}_{2}\right]^{+}$, where $\mathrm{G}$ denotes glycerol). The EH extractor potential was between -1.0 and $-2.0 \mathrm{kV}$, and the collector was at ground potential.

The EHMS spectra reported here represent the average of at least three magnet scans. Here and throughout this work, reported uncertainties represent the standard deviation of the mean $\left(s / n^{1 / 2}\right)$ of $n$ replicate measurements. Estimated uncertainties in derived values (e.g., percent ion pairing) were determined by standard propagation of errors analysis [35].

Solutions were degassed prior to analysis for at least $8 \mathrm{~h}$ at low heat $\left(50^{\circ} \mathrm{C}\right)$ under vacuum $\left(10^{-3}\right.$ torr $)$. In most EHMS experiments, sodium salts of various anions ( $\mathrm{NaA}$ ) were used as supporting electrolyte to maintain the total ionic strength at about $4.5 \mathrm{~mol} \%$ (glycerol $=100 \%$ ), as required for stable EH emission [33]. An internal standard of concentration comparable to the analyte was also included to facilitate comparison of sensitivity. In selecting a standard, one that does not ion pair would be optimum, but such a substance probably does not exist. Potassium was chosen because it, like sodium, does not associate extensively with anions in water [36]; presumably, its pairing in glycerol is also relatively low (compared to other cations). Accordingly, solutions included $0.2-0.3 \mathrm{~mol}$ $\%$ of the appropriate potassium salt (KA) as an internal standard; ions with peaks at $m / z=223$ and 315 $\left(\left[\mathrm{K}+\mathrm{G}_{2}\right]^{+}\right.$and $\left[\mathrm{K}+\mathrm{G}_{3}\right]^{+}$, respectively) were monitored as the reference ions. Diquat and quat analyte concentrations were about $0.1 \mathrm{~mol} \%$.

\section{Fast Atom Bombardment Mass Spectrometry}

Fast atom bombardment spectra were obtained with a VG ZAB-EQ mass spectrometer operating at an accelerating potential of $8 \mathrm{kV}$ with a mass resolution of at least 1000 (10\% valley definition). Research grade xenon (MG Industries) was used with an Ion Tech atom gun operating with $1.0 \mathrm{~mA}$ emission current and 8.0 $\mathrm{keV}$ energy.

Fast atom bombardment spectra were obtained from a thin film $(5-20 \mu \mathrm{L})$ of standard analyte solution applied to a modified [2] FAB probe or to a VG "Wobble" FAB probe. Results reported are the average of at least five normal magnet scans. Diquat solutions used for FAB/MS contained $0.1 \mathrm{~mol} \% \mathrm{NaA}$ and $0.001 \mathrm{~mol} \%$ analyte, much lower than the corresponding concentrations in the EH studies. This was necessary because the diquat was not detectable in FAB spectra obtained with solutions used for EHMS; analyte was evidently suppressed by the strong electrolyte signal. Conversely, EHMS sensitivity was not sufficient to detect $0.001 \mathrm{~mol}$ $\%$ diquat. Furthermore, the conductance of a $0.1 \mathrm{~mol}$ $\% \mathrm{NaA}$ solution is too low for stable $\mathrm{EH}$ emission [33]. Possibly because the quat is more surface-active than the diquat, it could be detected in FAB spectra of solutions with high electrolyte concentration. Fast atom bombardment mass spectrometry quat spectra were therefore obtained at both high $(0.1 \mathrm{~mol} \%$, with 4.5 $\mathrm{mol} \%$ electrolyte) and low $(0.001 \mathrm{~mol} \%$, with $0.1 \mathrm{~mol}$ $\%$ electrolyte) concentrations, with no difference in the trend of sampling efficiencies ( $S$; see below) for solutions of the various anions. In the diquat solutions, the ion at $m / z=207\left(\left[\mathrm{Na}+\mathrm{G}_{2}\right]^{+}\right)$was used as an internal reference ion. In the quat solutions, this and other electrolyte ions were suppressed (possibly due to the surface activity of the quat $[14,15])$. Thus, $\mathrm{Cs}^{+}$from dry $\mathrm{CsI}\left(C_{\rho}=1\right.$ in eq 2 , below on one of the targets of the "Wobble" FAB probe was used as an external standard, with quat solution applied to the other target. In these quat studies, five scans of the standard were obtained, and then the probe was shifted and five scans of the analyte were obtained. A single value of $S$ (see Results section) was then calculated from averages of these sets. Reported $S$ values represent averages of at least three replicates of this process. The solutions were not degassed before analysis because no spectral differences were observed when representative degassed and undegassed spectra were compared.

\section{Reagents}

Tetrabutylammonium bromide (reagent grade) was obtained from Eastman Chemicals and was dried at $110^{\circ} \mathrm{C}$ before use. Salts used as supporting electrolytes and standards (reagent grade) were obtained from Aldrich. The diquaternary ammonium diiodide was synthesized and recrystallized by standard methods [37]. Glycerol (Sigma grade) was obtained from Sigma.

\section{Results and Discussion}

To assess the counterion dependence of analyte sensitivity and the extent of ion pairing in EHMS, EH mass spectra of the diquaternary ammonium salt (I) were obtained in the presence of various sodium salt supporting electrolytes.

$$
\left(\mathrm{CH}_{3}\right)_{3} \stackrel{+}{\mathrm{N}}\left(\mathrm{CH}_{2}\right)_{5} \stackrel{+}{\mathrm{N}}\left(\mathrm{CH}_{3}\right)_{3} \cdot 2 \mathrm{I}^{-}
$$




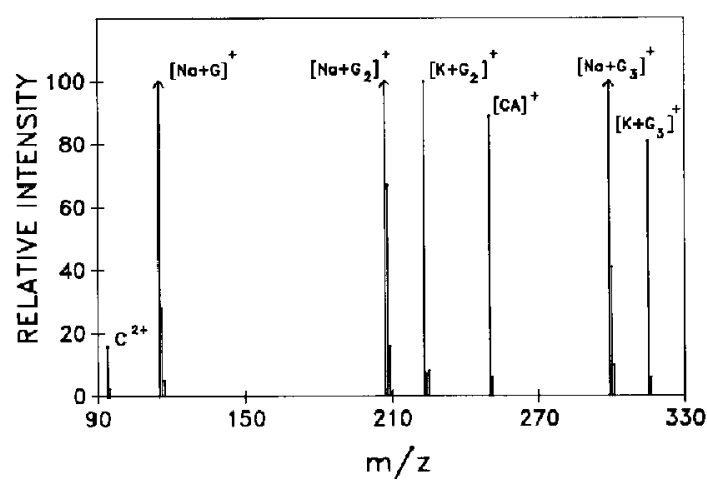

Figure 1. Electrohydrodynamic mass spectrum of a glycerol solution containing $4.5 \mathrm{~mol} \% \mathrm{NaNO}_{3} .0 .2 \mathrm{~mol} \% \mathrm{KNO}_{3}$, and 0.1 $\mathrm{mol} \%$ diquaternary ammonium iodide (I) (G denotes glycerol; $\mathrm{C}$ denotes diquat molecular cation; $A$ denotes nitrate). Intensities are relative to $\left[K+G_{2}\right]^{+}$, with sodium clusters off-scale.

Results with $\mathrm{NaNO}_{3}$ (Figure 1) are representative of all salts; only two ions attributable to the analyte were detected: doubly charged cation $\left(\mathrm{C}^{2+}\right)$ and the ion-paired species $\left(\mathrm{CA}^{+} ; \mathrm{A}=\mathrm{NO}_{3}^{-}\right.$for Figure 1$)$.

From the relative intensities of these ions, a measure of the percent ion pairing $(\% P)$ can be calculated:

$$
\% P=\frac{I_{\mathrm{CA}^{+}}}{I_{\mathrm{CA}^{+}}+I_{\mathrm{C}^{2+}}} \times 100
$$

where I denotes the average intensity of the subscripted ion. Because the appearance of EH mass spectra depends on solution chemistry [33], it is reasonable to assume that $\% P$ reflects the extent of ion pairing in solution. However, even if this is true (which seems likely; see discussion below), it is important to note that EHMS sensitivity to $\mathrm{CA}^{+}$is likely to be much greater than the sensitivity to $\mathrm{C}^{2+}[9]$. As a result, $\% P$ probably overestimates the relative abundance of ion pairs in solution.

For the seven ions tested, the trend in $\% P$ (Table 1) correlates with both gas-phase acidity and enthalpy of hydration (Table 2). Careful consideration of these correlations can provide insight into the relative importance of gas-phase and solution-phase chemistry in EHMS.

Reviewing gas-phase acidities, it is evident that $\mathrm{HClO}_{4}$ is the strongest gas-phase acid, so that $\mathrm{ClO}_{4}{ }^{-}$is the weakest gas-phase base. If gas-phase chemistry predominates, one would therefore predict that the perchlorate anion should be the least strongly ion paired. This is clearly inconsistent with the data of Table 1.

Similar reasoning could lead to a comparable conclusion that the trends in $\% P$ and enthalpy of hydration are contradictory, as follows. The small enthalpy of hydration for the perchlorate anion indicates that it has relatively weak interactions with po-
Table 1. Percent ion pairing (\%P) and sampling efficiencies $(S)$ for the diquat in solutions containing various anions ${ }^{\mathrm{a}}$

\begin{tabular}{lcrr}
\hline Anion (A) & $\begin{array}{c}\% P \\
(E H M S)^{\mathrm{b}}\end{array}$ & $\begin{array}{c}S \times 100 \\
(\text { EHMS) }\end{array}$ & $\begin{array}{c}S \times 100 \\
(\mathrm{FAB})^{\mathrm{b}}\end{array}$ \\
\hline Acatate & $12 \pm 1$ & $40 \pm 3$ & $6.3 \pm 0.4$ \\
Nitrite & $43 \pm 2$ & $50 \pm 2$ & $7.0 \pm 0.6$ \\
Chloride & $81 \pm 1$ & $84 \pm 2$ & $7.9 \pm 0.4$ \\
Bromide & $84 \pm 2$ & $97 \pm 4$ & $11.0 \pm 0.7$ \\
Nitrate & $85 \pm 2$ & $117 \pm 5$ & $15.4 \pm 0.7$ \\
& $59 \pm 1^{\mathrm{d}}$ & $74 \pm 2^{\mathrm{d}}$ & \\
lodide & $89 \pm 2$ & $122 \pm 4$ & $19.9 \pm 0.6$ \\
Perchlorate & $(95 \pm 2)^{\mathrm{e}}$ & $(134 \pm 5)^{\mathrm{B}}$ & $23.6 \pm 0.9$ \\
& $66 \pm 1^{\mathrm{d}}$ & $85 \pm 3^{\mathrm{d}}$ &
\end{tabular}

- See text for definitions.

b $[\mathrm{NaA}]=4.5 \mathrm{~mol} \%$, except where noted; [diquat $=0.1 \mathrm{~mol} \%$.

- $[\mathrm{NaA}]=0.1 \mathrm{~mol} \%$; [diquat $]=0.001 \mathrm{~mol} \%$

d $[\mathrm{NaA}]=2.0 \mathrm{mal} \% ;$ [diquat] $=0.1 \mathrm{~mol} \%$.

- Estimated value. $\mathrm{NaClO}_{4}$ was not soluble at the $4.5 \mathrm{~mol} \%$ level used for EHMS of the other supporting electrolytes. Because \%P should increase with electrolyte concentration (see data for nitrate), the value at $4.5 \mathrm{~mol} \%$ for $\mathrm{NaClO}_{4}$ was derived by comparison with $\mathrm{NaNO}_{3}$ samples at 2.0 and $4.5 \mathrm{~mol} \%$ using

$$
\% P_{\mathrm{NaClO}_{4}, 4.5 \mathrm{~mol} \%}=\% P_{\mathrm{NaClO}}, 2.0 \mathrm{~mol} \% \times \frac{\% P_{\mathrm{NaNO}_{3}, 4.5 \mathrm{~mol} \%}}{\% P_{\mathrm{NaNO}_{3}, 2.0 \mathrm{~mol} \%}}
$$

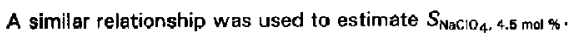

lar solvents (compared to the acetate anion, for example). By analogy, weak interactions with cations might be predicted. However, in contrast with the case for gas-phase acidities, there is an alternative perspective for comparing trends in $\% P$ and enthalpy of hydration. Solution-phase interactions represent a balance between Coulombic "ion pairing" and more complex ion-solvent interactions. The latter depend on such factors as polarizability and hydrogen bonding, accounting for the much wider variation (nearly a factor of 2) among hydration enthalpies, compared with the

Table 2. Enthalpy of hydration and gas-phase acidities for various anions

\begin{tabular}{lcc}
\hline Anion & Enthalpy of hydration & Gas-phase acidities $^{b}$ \\
\hline Acetate & -101 & $348.8 \pm 2.9$ \\
Nitrite & $-85^{\circ}$ & $338.2 \pm 4.3$ \\
Chloride & -87 & $333.4 \pm 0.2$ \\
Bromide & -80 & $323.5 \pm 0.1$ \\
Nitrate & -74 & $324.4 \pm 2.6$ \\
lodide & -71 & $314.4 \pm 0.1$ \\
Perchlorate & $-56^{\mathrm{d}}$ & $289.0 \pm 4.0^{\circ}$
\end{tabular}

- Enthalpy for $\mathrm{A}^{-}(\mathrm{g}) \rightarrow \mathrm{A}$ (aq) in $\mathrm{kcal} / \mathrm{mol}$, from ref. 38.

b Enthalpy for $\mathrm{HA}(\mathrm{g}) \rightarrow \mathrm{H}^{+}(\mathrm{g})+\mathrm{A}^{-}(\mathrm{g})$ in $\mathrm{kcal} / \mathrm{mol}$, from ref. 39 .

Estimate from ref. 40. Based on tabulated thermodynamic data [39, 41-43].

Estimate from ref. 40. Based on tabulated thermodynamic data [39, $41-43$ ) and an estimate for the gas-phase acidity (see footnote $\mathrm{e}$ ).

- Estimate based on MO calculations [44]. 
variation in gas-phase acidities $(\approx 20 \%$; Table 2$)$. Thus, it can be reasoned that with less competition from solvation, the perchlorate anion is better able to engage in Coulombic interactions, resulting in relatively extensive ion pairing. If this enhanced availability of $\mathrm{ClO}_{4}^{-}$ outweighs its intrinsically lower basicity (which seems likely in view of the small differences in basicity), the correlation is thereby explained. Such an explanation is consistent with the known sensitivity of EHMS to solution chemistry. By contrast, alternative explanations based on gas-phase interactions are contradicted both by the trends in acidity and by the low probability of field emission of anions from the positively biased $\mathrm{EH}$ emitter. We conclude that $\mathrm{ClO}_{4}^{-}$pairs most extensively of these seven ions in solution.

Turning now to the effect of this ion pairing on sensitivity, a measure of overall analyte sensitivity in the EHMS experiments can be derived from $S$, the relative sampling efficiency of the analyte:

$$
S=\frac{I_{\alpha} / C_{\alpha}}{I_{\rho} / C_{\rho}}
$$

where $I$ represents the average analyte $(\alpha)$ or reference $(\rho)$ intensity and $C$ represents the corresponding total concentrations. For the diquat, $I_{\alpha}$ refers to the sum of intensities of the doubly charged and ion-paired cations. As reasoned at the conclusion of the introduction to this paper, the direct correlation between $S$ and \%P in $\mathrm{EH}$ mass spectra of the diquat (Table 1) indicates that solvation plays a more important role in determining $S$ of the diquat than does variation in interactions with the extracting field. More evidence for the importance of solvation can be seen in spectra of metastable diquat ions. When the electrostatic analyzer potential is lowered to accept ions with less than full acceleration energy (i.e., ions experiencing energy loss due to in-flight desolvation), diquat sensitivity can be greatly enhanced as clusters containing the doubly charged cation and glycerol begin to dominate the EH mass spectrum [45]. This important role of solvation (relative to effects of varying ion-field interactions) suggests that even in the absence of an extracting field (e.g., in FAB), ion pairing may have a significant effect on analyte sampling efficiencies.

A direct comparison of \%P in EHMS and FAB/MS using the diquat was not possible owing to the negligible abundance of the doubly charged cation in FAB spectra. However, $S$ values based on the detectable $\mathrm{CA}^{+}$ions could be calculated with FAB data (Table 1). The FAB data reflect the trend observed in EHMS; the anion that pairs most in EHMS yields the highest diquat sampling efficiency by both techniques. Although the FAB sampling process is greatly complicated (relative to that of EHMS) by bombardment, the parallel ion-pairing behavior can most readily be interpreted as evidence for a solution chemical contribution to determining FAB sensitivity.
Table 3. Sampling efficiencies $(S)^{\text {a }}$ for the quat in solutions containing various anions

\begin{tabular}{lccc}
\hline Anion $(A)$ & $\begin{array}{c}S \\
\text { (EHMS) }^{\mathrm{b}}\end{array}$ & $\begin{array}{c}S \\
\text { (FAB/MS }^{\mathrm{b}}\end{array}$ & $\begin{array}{c}S \\
\text { (FAB/MS }^{\mathrm{c}}\end{array}$ \\
\hline Acetate & $168 \pm 18$ & $104 \pm 11$ & $58 \pm 2$ \\
Nitrite & $82 \pm 8$ & $48 \pm 5$ & $24 \pm 1$ \\
Chloride & $47 \pm 4$ & $33 \pm 3$ & $16 \pm 1$ \\
Bromide & $41 \pm 3$ & $27 \pm 1$ & $15 \pm 1$ \\
Nitrate & $38 \pm 4$ & $23 \pm 3$ & $13 \pm 1$ \\
& $53 \pm 5^{\mathrm{d}}$ & $44 \pm 3^{\mathrm{d}}$ & \\
lodide & $24 \pm 3$ & $13 \pm 2$ & $7 \pm 1$ \\
Perchlorate & $(19 \pm 2)^{\mathrm{a}}$ & $19 \pm 1)^{\mathrm{a}}$ & $5 \pm 1$ \\
& $26 \pm 3^{\mathrm{d}}$ & $17 \pm 2^{d}$ & \\
\hline
\end{tabular}

- See text for definition.

b $[\mathrm{NaA}]=4.5 \mathrm{~mol} \%$, except where noted: Iquat $=0.1 \mathrm{~mol} \%$.

- $[\mathrm{NaA}]=0.1 \mathrm{~mol} \% ;$ [quat $]=0.001 \mathrm{~mol} \%$.

d $[\mathrm{NaA}]=2.0 \mathrm{~mol} \%$; [quat $]=0.1 \mathrm{~mol} \%$

- Calculated as outlined in footnote e of Table 1.

As outlined above, for the more common case of singly charged analytes, neutralization by ion pairing would be expected to decrease $S$. As a test of this hypothesis [and to deconvolute possible contributions from the undetected (in FAB) doubly charged cation], FAB/MS and EHMS analyte sampling efficiencies were measured for the tetrabutylammonium (quat) cation in glycerol solutions with the various anions. Because this cation is structurally similar to the diquat, a similar anion dependence of ion pairing should pertain. Indeed, decreases in quat $S$ values mirrored trends of increased diquat ion pairing for both DI methods (Table 3).

One further observation is noteworthy on inspection of the data of Table 3. As described in the Experimental section, sensitivity differences necessitated that diquat EHMS and FAB/MS spectra be obtained at widely different concentrations. By contrast, FAB data for the quat could be obtained at both high and low concentrations. Comparison of the data for these two solutions (the last two columns in Table 3) reveals no difference in the overall qualitative trends, but a uniform quantitative difference: lower sensitivity for the lower concentration data. It is important to note that this is a change in sensitivity, not just a change in signal intensity, because the value of $S$ includes a correction for the concentration difference. However, since an external reference was used here, low sensitivities must result from reduced analyte intensities. Enhanced ion pairing at the lower concentration (due to the higher electrolyte/analyte ratio) is a likely cause.

\section{Conclusions}

The importance of solution chemistry in controlling the appearance of $\mathrm{EH}$ mass spectra has been well established. The use of anions that promote more extensive ion pairing will enhance the overall observation of multiply charged ions that otherwise interact strongly with 
the solvent but will reduce sensitivity to singly charged ions. These same solution properties also appear to be important in influencing analyte sensitivity in FAB/MS.

This is not to imply exclusive dependence; certainly some aspects of FAB ionization do depend on gasphase and other interactions. For example, it would be of interest to assess the strength of interactions responsible for the sensitivity gains observed when surfaceactive counterions were used with singly charged analytes in FAB $[14,15,25,27-31]$; surface enrichment evidently outweighs neutralization (if it occurs) in these systems. Tests for ion clustering using multiply charged analytes and singly charged surfactants could clarify factors contributing to this surfactant effect.

Despite these complications, the results of this study indicate that, in relatively simple systems, consideration of ion pairing may be useful in "designing" a FAB matrix. Interactions with effects of solution dielectric, inter alia, may complicate generalizations. However, in conjunction with compilations of matrix physical properties (e.g., ref. 46), consideration of data like those in Table 1 should facilitate efforts to rationalize matrix optimization.

\section{Acknowledgments}

This work was supported in part by grants from the National Science Foundation (DMR-8406825, cofunded by the Army Research Office; and CHE-8822787). The UTK Chemistry Mass Spectrometry Center is funded by the Science Alliance, a State of Tennessee Center of Excellence. The NSF Chemical Instrumentation Program also contributed to the acquisition of the ZAB-EQ (grant no. CHE-8609251). Helpful discussions with Dr. John Bartmess (University of Tennessee) are gratefully acknowledged.

\section{References}

1. De Pauw, E. Mass Spectrom. Rev. 1986, 5, 191.

2. Reynolds, J. D.; Cook, K. D. J. Am. Soc. Mass Spectrom., 1990, 1, 149-157.

3. Keough, T. Int. J. Mass Spectrom. Ion Processes 1988, 86, 155.

4. Miller, J. M.; Balasanmugam, K.; Nye, J.; Deacon, G. B.; Thomas, N. C. Inorg. Chem. 1987, 26, 560.

5. Kyranos, J. N,; duSorbier, B. M.; Wronka, J.; Vouros, P.; Kirby, D. P. Org. Mass Spectrom. 1988, 23, 443.

6. Musselman, B.; Watson, J. T. Biomed. Environ. Mass Spectrom. $1987,14,247$.

7. Williams, D. H.; Findeis, A. F.; Naylor, S.; Gibson, B. W. J. Am. Chem. Soc. 1987, 109, 1980.

8. Takayama, M.; Fukai, T,; Nomura, T. Int. J. Mass Spectrom. Ion Processes 1989, 89, R1.

9. Callahan, J. H.; Hool, K.; Reynolds, J. D.; Cook, K. D. Anal. Chem. 1988, 60, 714.

10. Busch, K. L.; Cooks, R. G. Science 1982, 218, 242.

11. Fraefel, A.; Seibl, J. Mass Spectrom. Rev. 1985, 4, 151.

12. Wysocki, V. H.; Kenttämaa, H. I.; Cooks, R, G. Int. J. Mass Spectrom. Ion Processes 1987, 75, 181.

13. Callahan, J. H.; Hool, K.; Reynolds, J. D.; Cook, K. D. Int. J. Mass Spectrom. Ion Processes 1987, 75, 291.
14. Ligon, W. V., Jr.; Dorn, S. B. Int. J. Mass Spectrom. Ion Processes $1985,63,315$.

15. Ligon, W. V., Jr.; Dorn, S. B. Int. J. Mass Spectrom. Ion Processes $1986,68,337$.

16. Wong, S. S.; Röllgen, F. W. Nucl. Instrum. Methods Phys. Res. 1986, B14, 436.

17. McCloskey, J. A.; Sethi, S. A.; Nelson, C. C. Anal. Chem. $1984,56,1975$.

18. Pelzer, G.; De Pauw, E.; Dung, D. V.; Marien, J. J. Phys. Chem. 1984, 88, 5065 .

19. Cerny, R. L.; Gross, M. L. Anal. Chem. 1985, 57, 1160.

20. Santana-Marques, M. G. O.; Ferrer-Correia, A. J. V.; Gross, M. L. Anal. Chem. 1989, 61, 1442.

21. Ashton, P. R.; Rose, M. E. Org. Mass Spectrom. 1986, 21, 388.

22. Brown, S. M.; Busch, K. L. Rapid Commun. Mass Spectrom. 1988, 2, 256.

23. Sunner, J. A.; Kulatunga, R.; Kebarle, P. Anal. Chem. 1986, 58, 2009.

24. Sunner, J. A.; Morales, A.; Kebarle, P. Anal. Chem. 1987, 59 , 1378.

25. De Pauw, E.; Pelzer, G.; Dung, D. V.; Marien, J. Biomed. Biophys. Res. Commun. 1984, 123, 27.

26. Hand, O. W.; Scheifers, S. M.; Cooks, R. G. Int. J. Mass Spectrom. Ion Processes 1987, 78, 131.

27. Ligon, W. V., Jr.; Dorn, S. B. Int. J. Mass Spectrom. Ion Processes 1987, 78, 99.

28. Ligon, W. V., Jr.; Dorn, S. B. Int. T. Mass Spectrom. Ion Processes 1984, 57, 75 .

29. Ligon, W. V., Jr.; Dorn, S. B. Int. J. Mass Spectrom. Ion Processes 1984, 61, 113.

30. Ligon, W. V., Jr.; Dorn, S. B. Anal. Chem. 1985, 57, 1993.

31. Ligon, W. V., Jr.; Dorn, S. B. Fresenius Z. Anal. Chem. 1986, 325,626 .

32. Busch, K, L.; Unger, S. E.; Vincze, A.; Cooks, R. G.; Keough, T. I. Am. Chem. Soc. 1982, 104, 1507.

33. Cook, K. D. Mass Spectrom. Rev. 1986, 5, 467.

34. Stimpson, B. P.; Evans, C. A., Jr. J. Electrostat. 1978, 5, 411.

35. Young, H. D. Statistical Treatment of Experimental Data; McGraw-Hill: New York, 1962; p 96.

36. Kleeberg, H.; Luck, W. A. P. J. Soln. Chem. 1983, 12, 369.

37. Ryan, T. M.; Day, R. J.; Cooks, R. G. Anal. Chem. 1980, 52, 2054.

38. Gibson, N. A.; Weatherburn, D. C. Anal. Chim. Acta 1972, $58,159$.

39. Lias, S. G.; Bartmess, J. E.; Liebman, J. F.; Holmes, J. L.; Levin, R. D.; Maliard, W. G. J. Phys, Chem. Ref. Data 1988, 17.

40. Bartmess, J. E., Univ. Tennessee, personal communication, 1989.

41. Wagman, D. D.; Evans, W. H.; Parker, V. B.; Schumn, R. H.; Halow, I.; Bailey, S. M.; Churney, K. L.; Nuttac, R. L. J. Phys. Chem. Ref. Data 1982, 11.

42. Klots, C. E. J. Phys. Chem. 1981, 85, 3585 .

43. Benson, S. W. Thermochemical Kinetics, 2nd ed.; Wiley: New York, 1976.

44. Koppel, I.; Pikver, R.; Sügis, A.; Suurmaa, E.; Lippmaa, E. Org. Reactivity 1981, 18, 1.

45. Cook, K. D.; Chan, K. W. S. Int. I. Mass Spectrom. Ion Processes 1983, 54, 135.

46. Cook, K. D.; Todd, P. J.; Friar, D. H. Biomed. Environ. Mass Spectrom. 1989, 18, 492. 